\title{
Self-assembled quantum dots: A study of strain energy and intersubband transitions
}

\author{
Yih-Yin Lin a) and Jasprit Singh \\ Department of Electrical Engineering and Computer Science, University of Michigan, Ann Arbor, \\ Michigan 48109-2122
}

(Received 29 April 2002; accepted 26 August 2002)

\begin{abstract}
In this article we examine the strain energy and intersubband optical transitions in self-assembled dots on GaAs and InP substrates. On the GaAs substrate, in addition to the InAs/GaAs dots we examine strain compensated InAs/GaAsP dots on GaAs substrates. We find that the strain energy configuration profile shows that there is preference for certain dot sizes and shapes. Our calculated dot sizes agree well with experimental observations. We find that the addition of phosphorus in the covering matrix reduces the total strain energy of the system with little effects on the intersubband transition strength for the vertical incident light. The reduced strain energy should allow one to incorporate a large number of dot array stacks for devices such as lasers and detectors and thus increases the optical responses. Our studies for the InAs/InP system show that due to the lower strain mismatch there is no particular preference for dot sizes. The optical response for intersubband transitions is weaker and occurs at longer wavelengths in comparison to the InAs/GaAs dots. (C) 2002 American Institute of Physics. [DOI: 10.1063/1.1515124]
\end{abstract}

\section{INTRODUCTION}

It is well known that the performance of many electronic and optoelectronic devices improves as system dimensionality is lowered. Additionally, lower dimensional systems can offer physical effects not present in higher dimensions. For these reasons there has been intense research in quantum wells, quantum wires, and quantum dots. Self-assembled quantum dots have emerged as a key technique to fabricate quasi-zero-dimensional systems. Semiconductor heterostructures containing quantum dots have been widely studied for making optoelectronic devices such as interband lasers, ${ }^{1}$ intersubband long wavelength detectors, ${ }^{2}$ and electro-optic modulators. ${ }^{3}$ Such structures can be realized using self assembly of strained structures by means of the StranskiKrastanov growth mode. The strain in the film growth plays a key role in determining the size uniformity and the spatial ordering of the deposited materials. The strain distribution in the quantum dot and its surrounding barrier also has a crucial influence on the electronic structure and optical properties.

It has been observed experimentally that self-assembled dots have a certain distribution of dot sizes which influence the photoluminescence linewidth. The linewidth, which is an order of magnitude larger than that seen in quantum wells, is due to inhomogeneous effects arising from a nonuniform distribution of dots formed during self assembly. Experimental studies have also shown that if InGaAs/GaAs (or other strained materials) layers are stacked on top of each other, dots align themselves over each other in the growth direction. ${ }^{4,5}$

It is important to have a series of vertical stacks of dots for optoelectronic devices since the optical response of a single layer of dots is too weak for most applications. How-

${ }^{a)}$ Electronic mail: yylin@engin.umich.edu ever, since there is a large strain in the dots, placing many dot layers can create a very high strain energy and perhaps dislocation formation. As a result there are interests in strain compensated quantum dots to lower the total strain energy of the system, but to preserve the low dimensional properties of the system. As an example, one can consider the growth of InAs on a GaAs substrate but to use GaAsP as the barrier matrix material. In this case InAs dots will be in compressive strain while GaAsP will be under tensile strain.

In this article we will address a number of issues related to dot strain energy and optical transition strengths. In particular we will address the following strain related issues:

How does the strain energy (per atom) vary in an InAs/ GaAs dot as we alter the dot size or shape? The answer to this may reveal how certain dot sizes are selected during self assembly.

How does the strain energy behave in strain compensated self-assembled dots? We will examine InAs/GaAsP dots matched to a GaAs substrate.

An important benefit of a quantum dot structure over a quantum well structure is the normal incident intersubband transition strength. In quantum wells this transition is forbidden in the conduction band subbands. However in quantum dots the vertical incident optical transition is quite strong. We will show results for intersubband optical transition in InAs/ GaAs and strain compensated quantum dots.

In addition to GaAs substrate based quantum dots we will also examine properties of InP based quantum dots. In this system the strain energy (between InAs and InP) is smaller $(\epsilon=-3.3 \%)$.

In Sec. II we will describe how the strain tensor and strain energy is calculated as a function of the dot size/shape or composition and how the electronic and optical transition are determined. 


\section{FORMALISM}

\section{A. Strain model and numerical solution}

The analysis of strain distribution in self-assembled quantum dots has been carried by several approaches. One approach is based on the Green's function, which assumes elastic isotropy and uniform elastic properties in both quantum dots and in the capping matrix to obtain a simple analytical result, but it fails to accurately determine the strain distribution due to the anisotropy of QD shapes. ${ }^{6,7}$ Numerical techniques such as finite element or finite difference methods are often employed to analyze the continuum mechanics. ${ }^{8} \mathrm{~A}$ symmetric spatial geometry is assumed in the calculation; however, the results can only reveal the shape geometry but not the details of structural sizes. Another popular approach is the atomistic simulation, where arbitrary geometries and empirical elastic properties can be taken into account. The solution is obtained as the system energy is minimized. In our approach, we use an atomistic Monte Carlo simulation and the valence force field (VFF) model developed by Keating ${ }^{9}$ and Martin $^{10}$ to calculate the strain distribution. This model has been proven to be successful in predicting and fitting the elastic constants of continuum theory and determining the atomic positions and strains in the QD structure.

Given a distribution of atoms on the mixed sublattices, the microscopic elastic energy $U$ is described by the valence force field model as

$$
\begin{aligned}
U= & \frac{1}{4} \sum_{i}\left[\sum_{j} \frac{3}{4} \alpha_{i j} \frac{\left(\mathbf{r}_{i j} \cdot \mathbf{r}_{i j}-d_{i j}^{2}\right)^{2}}{d_{i j}^{2}}\right] \\
& +\frac{1}{2} \sum_{i}\left[\sum_{j} \sum_{k \neq j} \frac{3}{4} \beta_{i j k} \frac{\left(\mathbf{r}_{i j} \cdot \mathbf{r}_{i k}+d_{i j} d_{i k} / 3\right)^{2}}{d_{i j} d_{i k}}\right]
\end{aligned}
$$

where $i$ represents all atomic sites, and $j, k$ are the nearest neighbors of $i . \mathbf{r}_{i j}$ denotes the vector from the $i$ th atom towards its $j$ th neighbor, and $d_{i j}$ is the strain-free equilibrium bond length in the binary constituents. $\alpha_{i j}$ and $\beta_{i j k}$ are the bond-stretching and bond-bending constants, respectively. For the bond-bending parameters, we take geometrically averaged $\beta_{i j k}=\sqrt{\beta_{i j} \beta_{i k}}$ following Ref. 11 .

In this article, we have two quantum dot systems: InAsGaAs and InAs-InP. A schematic view of the computational cell shown in Fig. 1 consists of a pyramidal-shaped dot with a square base on the top of a wetting layer. It is experimentally reported that the critical wetting layer thickness is 1.7 ML in the InAs/GaAs system ${ }^{12}$ and 2.1 MLs in the InAs/InP system. ${ }^{13}$ The orientations of the substrate material vary for different experiments. Here, we consider a typical GaAs(001) substrate for the InAs/GaAs case and InP(311) for the InAs/InP system. This orientation for InP is chosen since there are experimental studies reported for such dots. ${ }^{13,14}$

To shed light on how the strain is involved in the morphological evolution and size uniformity of QDs, we perform a quantitative study of the strain energy. In this model the interaction parameters for each atomic site can be related to the force constants for the material. In the past this approach

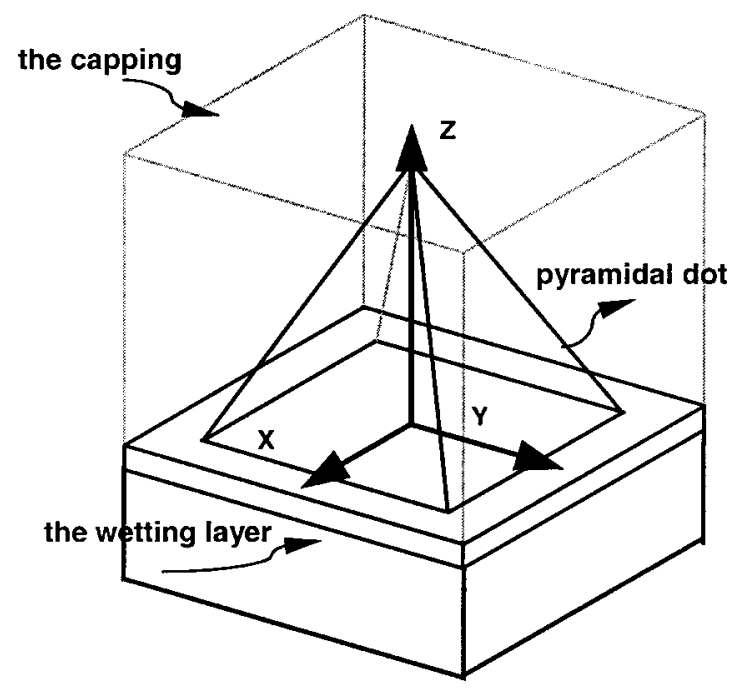

FIG. 1. Schematic illustration of the model used for the Stranski-Krastanow growth mode of the InAs pyramid and wetting layer.

has been applied to the dot structures where the covering material is also present. In this study we examine "the uncovered dots" as well since the dot size and shape are expected to be chosen via the energy of the uncovered surface. To simulate the uncovered dots we simply reduce the interaction parameters of the covering layer to negligible values. In Table I we list the parameters used in our study.

The strain tensors are obtained by minimization of the total energy within the framework of the VFF model. We start from an arbitrary choice of the atomic positions and minimize the system energy given by Eq. (1). Minimization of the total energy requires one to solve a coupled set of equations with $3 \mathrm{~N}$ variables, where $\mathrm{N}$ is the total number of atoms. Direct solution of these equations is impractical in our case since the system can contain more than 100000 atoms. We use the approach taken by several authors ${ }^{15-17}$ which has been shown to be quite efficient. In the beginning of the simulation all the atoms are placed on the GaAs lattice. We allow atoms to deviate from the starting position and assume periodic boundary conditions in the plane perpendicular to the growth condition. In each process, only one atom is displaced while other atoms are held at fixed positions. The displacement of that atom is determined by solving three linearized equations $\partial U / \partial x_{i}=0$, where $x_{i}$ is the displacement in the $x, y$, or $z$ direction. All the atoms are displaced in sequence. The whole sequence is repeated until the maximum distance moved in the sequence is so small that there is essentially no change in the system energy.

To calculate the configuration energy diagram we calculate the minimum energy configuration for a number of dot sizes and shapes. We limit our studies to pyramidal dots

TABLE I. Constants used to calculate the VFF elastic energy from Ref. 10

\begin{tabular}{lrrrc}
\hline \hline & GaAs & InAs & InP & GaP \\
\hline$\alpha(\mathrm{N} / \mathrm{m})$ & 41.19 & 35.18 & 43.04 & 47.32 \\
$\beta(\mathrm{N} / \mathrm{m})$ & 8.95 & 5.50 & 6.24 & 10.44 \\
\hline \hline
\end{tabular}


since experimental evidence suggests that this shape is usually chosen. In principle, however, our model can calculate the strain energy for any shapes.

\section{B. Electronic spectrum}

Once we have the strain tensors for the InAs dots of various sizes, we can use the strain-modified eight-band $\mathbf{k} \cdot \mathbf{p}$ method, where the influence of remote bands on the conduction and valence bands is included, to calculate the electronic spectra. The details are described in Ref. 18. The band lineup for a strained structure at the Brillouin zone center is determined following Ref. 19. Both the conduction and valence band states can be derived using the eight-band Hamiltonian. We use a finite-difference method to solve this problem numerically. Since the typical matrix sizes needed are very large $\sim 10^{5} \times 10^{5}$, we use the Arnoldi/Lanczos method to solve the eigenvalue problem.

\section{Intersubband optical spectra}

As noted in Sec. I, intersubband optical transitions for the conduction band distinguish quantum dots from the wells. We examine intersubband optical absorption for the various dots studied in this article. The intersubband absorption coefficient $(\alpha)$ of a photon with energy $\hbar \omega$ in a quantum dot layer is

$$
\alpha(\hbar \omega)=\frac{\pi e^{2} \hbar}{\varepsilon_{0} n_{0} c m_{0}^{2} V_{a v}} \frac{1}{\hbar \omega} \sum_{f i}\left|\mathbf{a} \cdot \mathbf{p}_{f i}\right| N(\hbar \omega),
$$

where $c$ is the speed of light, $\varepsilon_{0}$ is the dielectric constant of vacuum, $n_{0}$ is the refractive index, and $V_{a v}$ is the average dot volume. $\mathbf{a}$ is the polarization of the light, $\mathbf{p}_{f i}$ is the momentum matrix element between the initial $i$ and final $f$ states, and $N(\hbar \omega)$ is the electron density of states. We assume that the ground state in the dots is a very sharp, almost deltafunction peak. In reality this peak is broadened by both homogeneous and inhomogeneous effects. Due to the large fluctuation of dot sizes, inhomogeneous broadening is significantly larger than homogeneous broadening; thus we only consider inhomogeneous broadening. We take the broadening to be Gaussian so that

$$
N(\hbar \omega)=\frac{1}{\sqrt{2 \pi} \sigma} \exp \left[\frac{\left(E_{f i}-\hbar \omega\right)^{2}}{(2 \sigma)^{2}}\right]
$$

where $E_{f i}$ is the energy separation between states $f$ and $i$, and $\sigma$ is the linewidth of the transition, which is taken as $30 \mathrm{meV}$ in the simulation.

In the eight-band model, each wave function is the sum of contribution from each of the eight bands:

$$
\psi_{n}=\sum_{j=1}^{8} \phi_{n j}(\mathbf{r}) u_{j},
$$

where $\phi_{n j}$ is the envelope function of state $n$ and band $j$, and $u_{j}$ is the central-cell part. The momentum matrix element for the transition from state $\psi_{i}$ to state $\psi_{f}$ is then given by

$$
\begin{aligned}
p_{f i} & =\left\langle\psi_{f}|\mathbf{p}| \psi_{i}\right\rangle \\
& =\sum_{j, k}\left\langle\phi_{f i} \mid \phi_{i k}\right\rangle\left\langle u_{i} \mid u_{k}\right\rangle+\sum_{j}\left\langle\phi_{f j}|\mathbf{p}| \phi_{i j}\right\rangle .
\end{aligned}
$$

Because there is considerable band mixing in the quantum dots, the first summation term is nonzero as is often the case in the intersubband transitions in quantum wells. This term is quite large and must be included.

\section{RESULTS}

This section contains two classes of results. In the first part, we address structural issues for InAs dots grown on the GaAs and InP substrate. We present the configuration energy for several different dot sizes. For the InAs/GaAs system, we also examine the strain compensation in InAs QDs covered by GaAsP matrix. In the second part, we show the results for the intersubband absorption spectra for InAs dots on InP substrate and on GaAs substrate with GaAsP capping.

Using the VFF model we can calculate the strain energy for various chosen dot shapes and sizes. Since the dots can be chosen in infinite ways we select our dots to pyramidal shapes. In our simulations we alter the dot height for a given base to height ratio and for a given height we vary that ratio. We also assume a wetting layer thickness of one monolayer for the InAs/GaAs system. The simulation results are for (001) GaAs substrates and for (311) InP substrates.

It is well known that if the InAs layer is to be deposited in an atomically flat layer on GaAs the layer will be under biaxial compression. The in-plane lattice constant will be forced to be the same as that of GaAs and the out-of-plane lattice constant will be larger due to the Poisson's effect. In such a configuration the strain energy is quite large $(\sim 12 \mathrm{meV}$ per atom). The reason for dot formation is that this strain energy is reduced when the InAs is in a dot form. We examine the InAs dot strain energy without the barrier covering material as a function of the dot height keeping the ratio of the height to base at 1:2. The results for the strain energy per atom are shown in Fig. 2. The results shown are for the dot region only. We see that the strain energy has a minimum at a height of $62 \AA$. As the dot size increases or decreases beyond this value the strain energy increases. However, the increase is not very sharp and is only $\sim 0.3 \mathrm{meV}$ per atom for an increase (decrease) of $\sim 10 \AA$. As a result we expect that there will be a distribution of InAs dots with sizes centered around $62 \AA$. The discussion above has been observed experimentally by Krishna et al. ${ }^{20}$ They report a distribution of InAs dots with the most probable size being $\sim 65 \AA$. We have also done studies in which the ratio of the dot basewidth to dot height is varied. We find that the energy is lowest for the 2:1 ratio.

Figure 2 shows how the transition energy $E_{1}^{e}-E_{1}^{h h}\left(E_{1}^{e}\right.$ and $E_{1}^{h h}$ being the ground states for electrons and holes in the dot) varies as a function of dot sizes. These results are based on our eight-band $\mathbf{k} \cdot \mathbf{p}$ calculations.

We now examine the InAs/InP dot system. The results shown in Fig. 3 as noted earlier are for a (311) substrate. In this system the strain is half of that in the InAs/GaAs system and thus the strain energy is consequently much smaller. In 


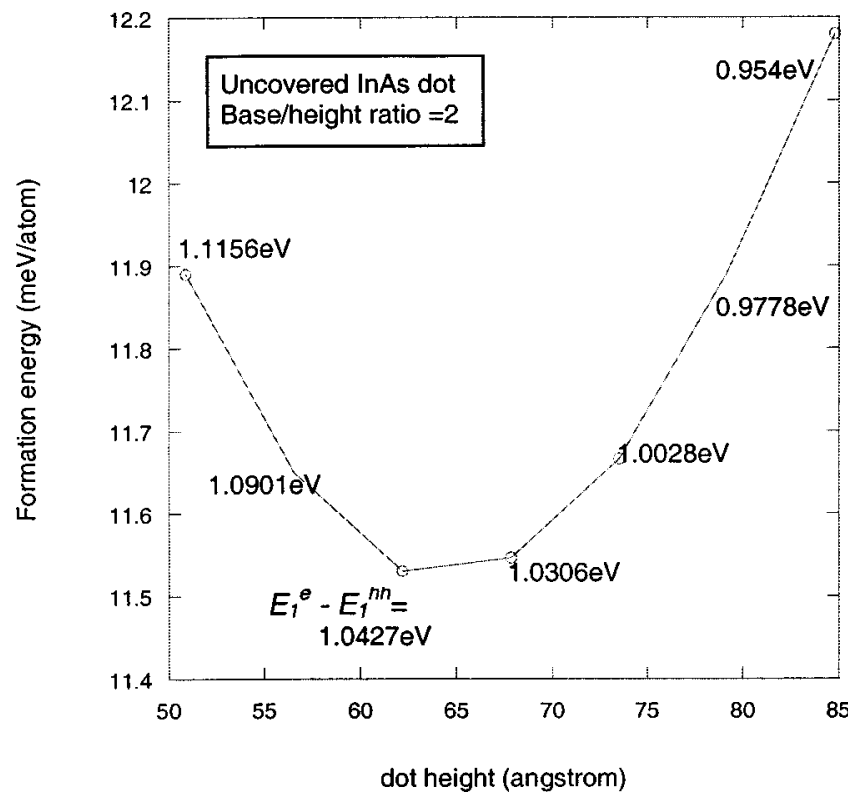

FIG. 2. Strain energy per atom for InAs dot grown on (001) GaAs substrate at various dot heights. Dotted lines represent the strain energy when the InAs wetting layer is included. The transition energy values from the ground states of holes to electrons $E_{1}^{e}-E_{1}^{h h}$ are also listed for each dot size.

contrast to the InAs/GaAs system case, here we do not see a clear minimum in the strain energy as a function of the dot size. In the figure also shown is the total strain energy per atom when the wetting layer region is included, and this energy has a minimum at dot height $59 \AA$. Experimental studies in the InAs/InP dots show a photoluminescence emission peak at around $0.78 \mathrm{eV}$, which should be for a dot height of $70 \AA$ according to our eight-band $\mathbf{k} \cdot \mathbf{p}$ simulation.

Experimental studies for InAs/InP dots show that there is a very nonuniform arrangement of dots in the self-assembled structure. The photoluminescence linewidth is also very

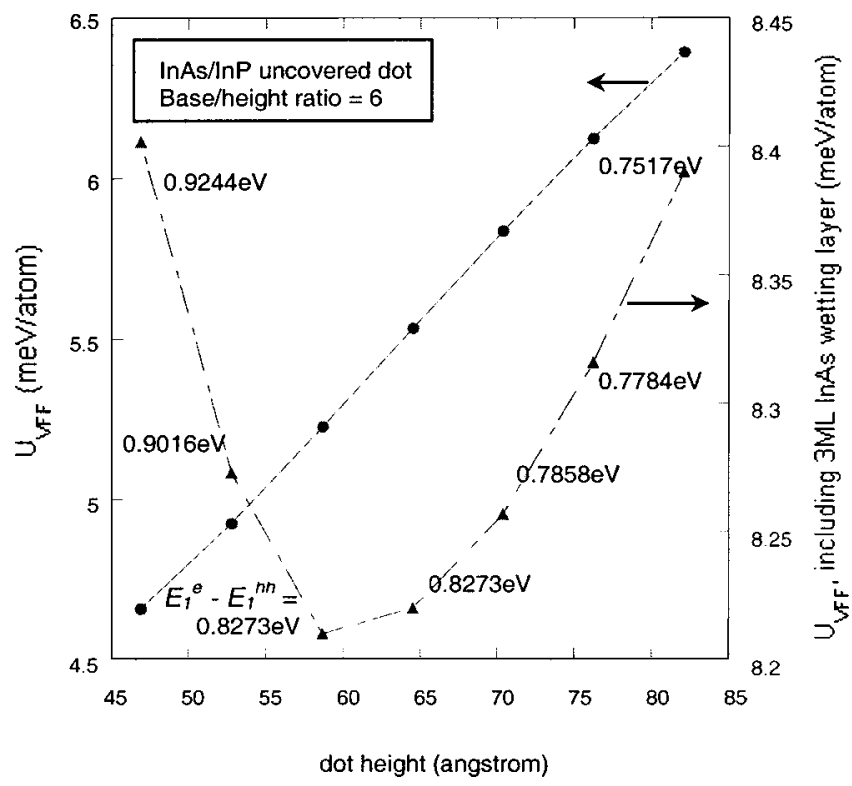

FIG. 3. Strain energy per atom for InAs dot grown on (311) InP substrate at various dot heights. The transition energy values $E_{1}^{e}-E_{1}^{h h}$ are also listed for each dot size. broad. In Fig. 3 the $E_{1}^{e}-E_{1}^{h h}$ values calculated show that even though the strain energy variation as a function of dot sizes is small these effective band gap (which determine the photoluminescence transition) varies considerably with dot size.

As our last strain study we examine InAs dots grown on a GaAs substrate but covered with GaAsP rather than GaAs. As noted in Sec. I the study is motivated by the strain balancing consideration. InAs in the dot is in compressive strain while GaAsP is under tensile strain. In this system if the InAs dot and the GaAsP matrix were completely mixed up randomly the resulting alloy could be lattice matched to GaAs with no strain energy. However, in the InAs/GaAsP dot there is strain energy present. We use the VFF model to calculate the strain energy and strain tensor in the InAs/ GaAs $1-x \mathrm{P}_{x}$ system as a function of GaP composition $x$.

In the quantum-well structure, the substrate lattice constant $a_{0}$ for balanced strain is a function of the elastic properties and thicknesses in tensile and compressive regions. Ekins-Daukes et $a l^{21}$ derived this relation from the continuum elastic model:

$$
a_{0}=\frac{t_{1} A_{1} a_{1} a_{2}^{2}+t_{2} A_{2} a_{2} a_{1}^{2}}{t_{1} A_{1} a_{2}^{2}+t_{2} A_{2} a_{1}^{2}},
$$

where the subscripts of 1 and 2 denote different layers on the substrate, $A=C_{11}+C_{12}-2 C_{12}^{2} / C_{11}$ with elastic stiffness coefficients $C_{11}$ and $C_{12}, a$ is the lattice constant, and $t$ is the thickness of each well. In the quantum dot case, it is more complicated since the dot itself is embedded by the barrier matrix and the geometry varies in two dimensions along the growth direction. In the InAs/GaAs system, it would be desirable if the lattice constant of the covering GaAsP material is small near the GaAs substrate but increases towards the InAs dot tip. In this study, we assume a uniform $\mathrm{GaAs}_{1-x} \mathrm{P}_{x}$ covering material and we can obtain a rough estimate of the desirable lattice constant of GaAsP using the equation given above by letting $a_{0}$ equal to $a_{\mathrm{GaAs}}$. To deal with the quantum dot structure, we divide the structure along the $z$-direction layer by layer, calculate the desirable lattice constant of the tensile strain region of each layer and take an average of them. For an InAs QD of $62 \AA$ height and $124 \AA$ width embedded in the $80 \AA$ GaAsP matrix at a dot density of 4 $\times 10^{11} \mathrm{~cm}^{-2}$, the lattice constant of the $\mathrm{GaAs}_{1-x} \mathrm{P}_{x}$ matrix material for tensile strain compensation is $5.5671 \AA$ at $x$ $=0.42$.

Figure 4 shows the strain energies in the dot region which is under compressive strain and in the covering matrix region which is under tensile strain. To achieve a strain-free environment, the strain energy under the compressive stress must be equivalent to that of the tensile stress. As seen in the figure, tensile strain increases as the gallium phosphorus composition $(x)$ grows and becomes equivalent to the compressive strain when $x \sim 0.4$. It is very close to the estimation discussed above. It should also be noted that as expected the compressive strain energy in the InAs dot region remains essentially unchanged as the composition of the covering layer is altered. The tensile strain energy in the covering matrix increases rapidly as the $\mathrm{P}$ composition is increased. 


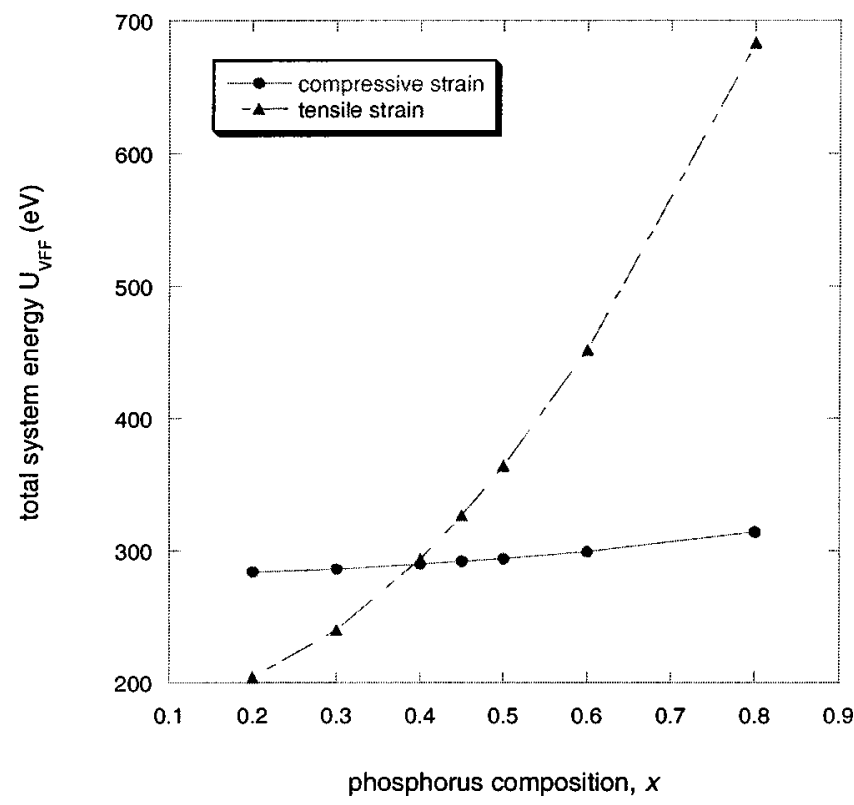

FIG. 4. Strain energy in the dot (compressive strain) and in the surrounding matrix (tensile strain) as a function of phosphorus composition of the $\operatorname{GaAs}_{1-x} \mathrm{P}_{x}$ matrix. The dot height is $62 \AA$ and the base/height ratio is $2: 1$. The dot is embedded in the $80 \AA$ matrix when the density is set to be 2.9 $\times 10^{11} \mathrm{~cm}^{-2}$. The system contains 4600 atoms in the compressive strain region, and 17270 atoms in the tensile strain region.

In Fig. 5 we illustrate the hydrostatic strain $\varepsilon_{x x}+\varepsilon_{y y}$ $+\varepsilon_{z z}$ (top panels) and biaxial strain $\varepsilon_{z z}-0.5\left(\varepsilon_{x x}+\varepsilon_{y y}\right)$ (lower panels) for InAs/GaAs, InAs/ $\mathrm{GaAs}_{0.5} \mathrm{P}_{0.5}$, and InAs/ $\mathrm{GaP}$ structures all on a GaAs substrate. The gradation of shadowing in the figures represent the strain values. Darkshaded regions represent high strain. We notice that the hydrostatic strain is very large in the dots with either no $\mathrm{P}$ or with $\mathrm{GaP}$ as the covering material. However InAs/ $\mathrm{GaAs}_{0.5} \mathrm{P}_{0.5}$ structures have quite low strain. Such structures would thus be desirable as dots for applications where a large number of dot stacks are needed. We find that the addition of phosphorus in the surrounding matrix reduces the overall strain energy considerably at the optimal phosphorus composition.
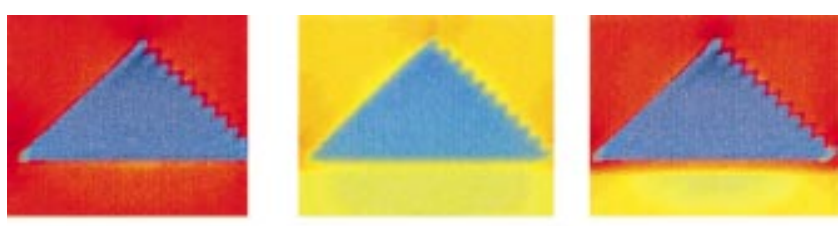

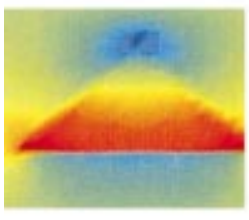

$x=0$

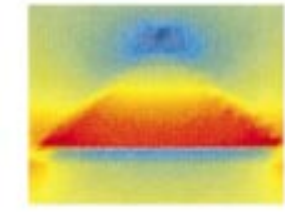

$x=0.5$

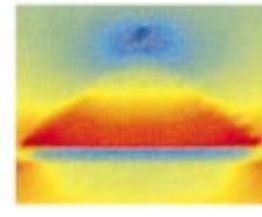

$x=1.0$
FIG. 5. (Color) Cross-section view along (001) direction of strain distribution in InAs/GaAs system for different $\mathrm{GaP}$ compositions in the capping layer region. Figures on the top show the hydrostatic strain. Below is the biaxial strain. The shading levels represent the strain values.
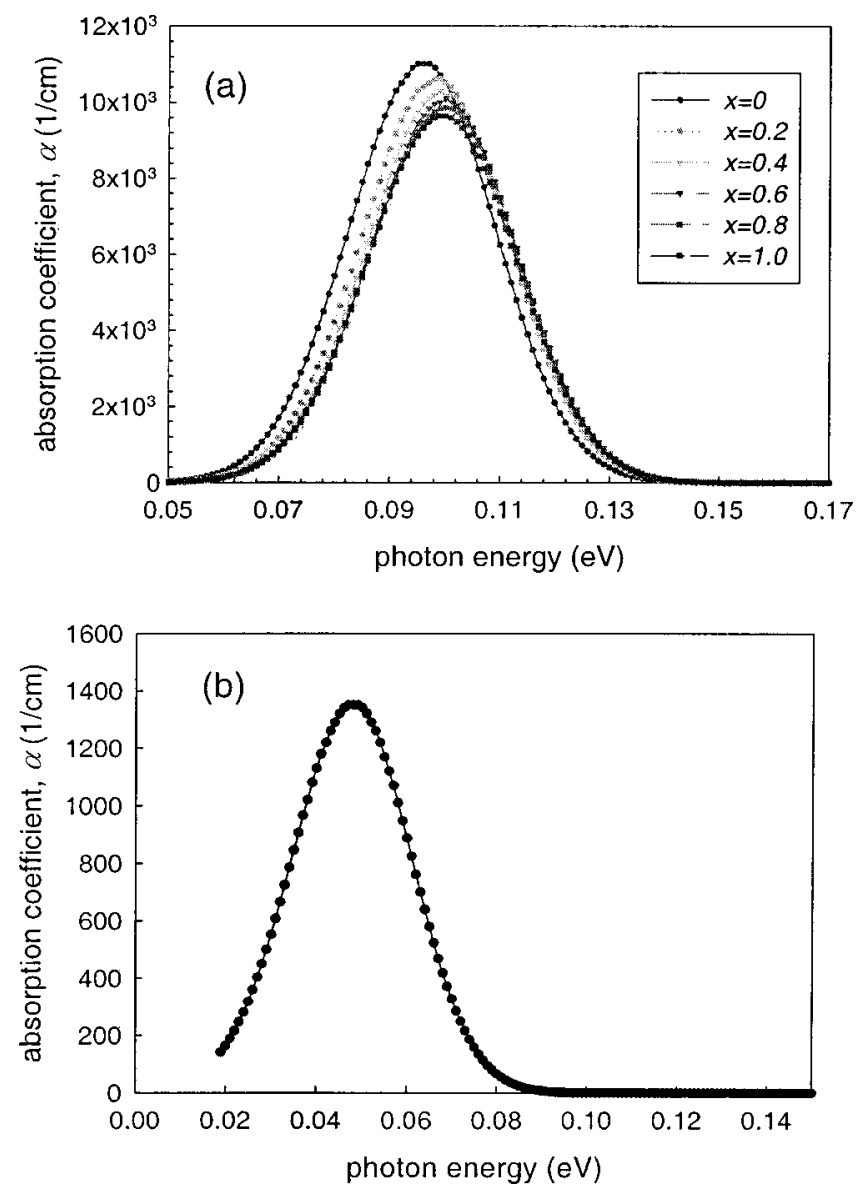

FIG. 6. Intersubband absorption spectra for vertical incident light for: (a) InAs/GaAs dots with various $\mathrm{GaAs}_{1-x} \mathrm{P}_{x}$ capping compositions, $x$, (b) InAs/ InP dots. The linewidth is taken to be $30 \mathrm{meV}$.

We now come to the next issue discussed in this article, how are the intersubband optical transitions influenced by strain and confinement in self-assembled dots? As noted in Sec. I, an important consequence of 3D confinement is that quantum dots have a strong intersubband absorption for vertical incident light. We present results for this transition in Fig. 6 for the different kinds of dots examined here. For the GaAs based dots we examine InAs dots with a GaAs matrix and GaAsP matrix with different P content as shown in Fig. 6(a). The optical transition strength is strongest for the GaAs matrix case. This is due to the high strain in the InAs dot which causes a stronger momentum matrix element. There is a slight increase in the intersubband energy due to the addition of the $\mathrm{P}$ in the matrix as can be expected from the increase in the confining energy of the barrier region.

In Fig. 6(b) we show results for the intersubband absorption spectrum for an InAs/InP dot of height $40 \AA$ and a base width of $240 \AA$. We see a weaker absorption coefficient for vertical incident light due to the larger base to height ratio $(6: 1)$ here. The intersubband separation is quite a bit smaller than that in the InAs/GaAs dot. Such dots may be useful for very long wavelength detectors. Our results show that vertical incident intersubband transitions are strong in all of the dots studied. 


\section{SUMMARY}

In this article we examined the strain energy issues and optical response of intersubband transitions in GaAs and InP based dots. We find that InAs dots grown on GaAs substrate have a strain driven preference in dot size. Such a preference is not found in the InP based dots. We find that the use of InAs dots with a GaAsP matrix reduces the strain energy in the system but still maintains a high intersubband absorption for vertical incident light. In the InAs/InP dot the intersubband absorption is quite strong and the transition energies are lowered to about $0.05 \mathrm{eV}$.

\section{ACKNOWLEDGMENT}

This article has been funded by the U.S. Army MURI Program.

${ }^{1}$ S. Krishna, O. Quasaimeh, P. Bhattacharya, P. J. MaCann, and K. Namjou, Appl. Phys. Lett. 76, 3355 (2000).

${ }^{2}$ H. Jiang and J. Singh, Physica E (Amsterdam) 2, 720 (1998).

${ }^{3}$ O. Qasaimeh, K. Kamath, P. Bhattacharya, and J. Phillips, Appl. Phys. Lett. 72, 1275 (1998).

${ }^{4}$ Q. Xie, A. Madhukar, P. Chen, and N. P. Kobayashi, Phys. Rev. Lett. 75, 2542 (1995).
${ }^{5}$ G. S. Solomon, J. A. Trezza, A. F. Marshall, and J. S. Harris, Jr., Phys. Rev. Lett. 76, 952 (1996).

${ }^{6}$ A. D. Andreev, J. R. Downes, D. A. Faux, and E. P. O'Reilly, J. Appl. Phys. 86, 297 (1999).

${ }^{7}$ M. Califano and P. Harrison, J. Appl. Phys. 91, 389 (2002).

${ }^{8}$ T. Benabbas, P. François, Y. Androussi, and A. Lefebvre, J. Appl. Phys. 80, 2763 (1996)

${ }^{9}$ P. N. Keating, Phys. Rev. 145, 637 (1966).

${ }^{10}$ R. M. Martin, Phys. Rev. B 1, 4005 (1969).

${ }^{11}$ M. Podgorny, M. T. Czyzyk, A. Balzarotti, P. Letardi, N. Motta, A. Kisiel, and M. Zimnal-Starnawska, Solid State Commun. 55, 413 (1985).

${ }^{12}$ M. Grundmann, J. Christen, N. N. Ledentsov, J. Böhrer, D. Bimberg, S. S. Ruvimov, P. Werner, U. Richter, U. Gösele, J. Heydenreich, v. M. Ustinov, A. Yu. Egorov, A. E. Zhukov, P. S. Kop'ev, and Zh. I. Alferov, Phys. Rev. Lett. 74, 4043 (1995).

${ }^{13}$ S. Fréchengues, N. Bertru, V. Drouot, B. Lambert, S. Robinet, S. Loualiche, D. Lacombe, and A. Ponchet, Appl. Phys. Lett. 74, 3356 (1999).

${ }^{14}$ K. Nishi, H. Saito, and S. Sugou, Appl. Phys. Lett. 74, 1111 (1999).

${ }^{15}$ J. Bernard and A. Zunger, Appl. Phys. Lett. 65, 165 (1994).

${ }^{16}$ F. Glas, J. Appl. Phys. 66, 1667 (1989).

${ }^{17}$ M. R. Weidmann and K. E. Newman, Phys. Rev. B 45, 8388 (1996).

${ }^{18}$ H. Jiang and J. Singh, Appl. Phys. Lett. 71, 3239 (1997).

${ }^{19}$ C. G. Van De Walle, Phys. Rev. B 39, 1871 (1989).

${ }^{20}$ S. Krishna, J. Sabarinathan, P. Bhattacharya, B. Lita, and R. S. Goldman, J. Vac. Sci. Technol. B 18, 1502 (2000).

${ }^{21}$ J. K. Ekins-Daukes, J. Zhang, D. B. Bushnell, K. W. J. Barnham, M. Mazzer, and J. S. Roberts, in Proceedings of the 28th IEEE PV Specialists Conference, IEEE, USA, 2000, pp. 1273-1276. 